\title{
Spectral Analyses of the [WCL]-type CSPN He 2-459, M 2-43, SwSt 1, PM 1-188 and IRAS 21282+5050
}

\section{U. Leuenhagen}

Institut für Astronomie und Astrophysik, Universität Kiel, D-24098 Kiel, Germany; (present address: I. Phys. Institut, Universität Köln, Zülpicher Str. 77, D-50937 Köln, Germany)

In this work recent results of spectral analyses of five late-type [WC] central stars are presented. We use spherically expanding model atmospheres to simulate non-LTE radiation transfer in mass-loosing envelopes. A detailed description of the model calculations and the stellar parameters of further eight [WCL]-CSPN can be found in Leuenhagen \& Hamann 1994, A\&A 283, 567, and Leuenhagen et al. 1996, A\&A 312, 167.

The spectral fit of the observations provides the effective temperature $T_{*}$, the final velocity $v_{\infty}$, the element abundances $\beta$ of $\mathrm{H}, \mathrm{He}, \mathrm{C}$ and $\mathrm{O}$ and the transformed radius $R_{\mathrm{t}}$ (see Table 1). The latter parameter is anticorrelated to the wind density. In the case of known reddening, visual brightness and distance, $R_{\mathrm{t}}$ can be disentangled into stellar radius $R_{*}$ and mass-loss rate $\dot{M}$. Note, that all results for $R_{*}, \dot{M}$ and $L$ given in Table 2 depend on the distance $\left(R_{*} \sim d, \dot{M} \sim d^{3 / 2}, L \sim d^{2}\right)$.

The most surprising result is the certain identification of hydrogen in PM 1-188 and IRAS 21282. The wind densities of SwSt 1 and IRAS 21282 are unusually small.

Table 1: Resulting stellar parameters. $T_{2 / 3}$ is related to $R\left(\tau_{\text {Ross }}=2 / 3\right), R_{\mathrm{t}}$ is defined as $R_{*}\left(v_{\infty} / \dot{M}\right)^{2 / 3}$ and the element abundances are given in mass fractions.

\begin{tabular}{lccccccccc}
\hline & subtype & $T_{*}$ & $T_{2 / 3}$ & $v_{\infty}$ & $R_{\mathrm{t}}$ & $\beta_{\mathrm{H}}$ & $\beta_{\mathrm{He}}$ & $\beta_{\mathrm{C}}$ & $\beta_{\mathrm{O}}$ \\
& & {$[\mathrm{kK}]$} & {$[\mathrm{kK}]$} & {$[\mathrm{km} / \mathrm{s}]$} & {$\left[R_{\odot}\right]$} & {$[\%]$} & {$[\%]$} & {$[\%]$} & {$[\%]$} \\
\hline He 2-459 & [WC8] & 77 & 52.6 & 1000 & 2.15 & $(<2)$ & 40 & 50 & 10 \\
M 2-43 & [WC8] & 77 & 53.5 & 850 & 2.19 & - & 40 & 50 & 10 \\
SwSt 1 & [WC9] & 37 & 36.5 & 400 & 25.91 & $(<10)$ & 43 & 52 & $5 ?$ \\
PM 1-188 & [WC11] & 35 & 31.7 & 360 & 7.07 & 1 & 42 & 50 & 7 \\
IRAS 21282 & [WC11] & 28 & 27.4 & 180 & 51.95 & 10 & 43 & 46 & 1 \\
\hline
\end{tabular}

(): affected by strong nebular lines; a: no spectra available; ?: uncertain

Table 2: Using photometry, extinction and distances from the literature the stellar radii $R_{*}$, the mass loss rates $\dot{M}$ and the luminosities $L$ can be determined.

\begin{tabular}{llllllll}
\hline & subtype & $\begin{array}{c}m_{\mathrm{V}} \\
{[\mathrm{mag}]}\end{array}$ & $\begin{array}{c}E_{\mathrm{B}-\mathrm{V}} \\
{[\mathrm{mag}]}\end{array}$ & $\begin{array}{c}d \\
{[\mathrm{kpc}]}\end{array}$ & $\begin{array}{c}R_{*} \\
{\left[R_{\odot}\right]}\end{array}$ & $\begin{array}{c}\log \dot{M} \\
{\left[M_{\odot} / \mathrm{yr}\right]}\end{array}$ & $\begin{array}{l}\log L \\
{\left[L_{\odot}\right]}\end{array}$ \\
\hline He 2-459 & [WC8] & 12.7 & 0.85 & 3.4 & 0.84 & -5.01 & 4.37 \\
M 2-43 & [WC8] & $15.7 ?$ & $1.26 ?$ & 1.4 & 0.22 & -5.96 & 3.21 \\
SwSt 1 & [WC9] & 11.9 & 0.40 & 1.4 & 1.03 & -6.90 & 3.27 \\
PM 1-188 & [WC11] & 14.6 & 0.83 & - & 1.88 & -5.70 & 3.70 \\
IRAS 21282 & [WC11] & 14.3 & 1.60 & $<2$ & $<3.12$ & $<-6.98$ & $<3.75$ \\
\hline
\end{tabular}

?: uncertain; PM 1-188: $L$ presumed (no distance estimation available) 